\title{
Sul teorema di unicità per le equazioni del campo elettromagnetico in un plasma (*).
}

Dario Grafel (Bologna) (**)

A Bruno Finzi nel suo 70mo compleanno

Sunto. - Si dimostrano alcuni teoremi di unicità per le equazioni non-lineari del campo elettromagnetico in un plasma in cui si tiene conto della pressione dei corpuscoli elettrici. I teoremi sono sempre dimostrati nel caso non stazionario, anzitutto supponendo che al plasma occupi tulto lo spazio, poi vengono estesi al caso ai un dominio finito con op. portune ipotesi alla frontiera. Infine si ottengono teoremi di unicità nel caso in cui, per effetto di campo magnetico, la pressione diventa anisotropa.

1. - Dimostrerò, in questa memoria, alcuni teoremi di unicità per le equazioni non lineari macroscopiche del campo elettromagnetico in un plasma in cui si deve tener conto della pressione esercitata dai corpuscoli elettrici (warm plasma).

Le equazioni in discorso, (1) supponendo per semplicita sensibili al campo elettromagnetico solo gli elettroni e trascurabili i termini dissipativi (del resto il tenerne conto non offrirebbe ulteriori difficoltal) sono le ordinarie equazioni di MaXwell:

$$
\begin{gathered}
\operatorname{rot} \boldsymbol{H}=\varepsilon \frac{\partial E}{\partial t}+N q v+J \\
\operatorname{rot} \boldsymbol{E}=-\mu \frac{\partial \boldsymbol{H}}{\partial t}
\end{gathered}
$$

in cui $\boldsymbol{E}$ e $\boldsymbol{H}$ indicano rispettivamente il campo elettrico e il campo magnetico, $v$ e $N$ la velocita media e il numero degli elettroni per unità di volume,

${ }^{*}$ ) Lavoro eseguito nell'ambito dell'attività dei gruppi di ricerea del C. $\mathbf{N}$ R.

(**) Entrata in Redazione il 19 gennaio 1970.

(1) Per queste equazioni si veda p.e. L. Oster, Linearised theory of plasma oseillations, Revue of Modern Physies XXXII (1060)-141- M. H. СонеN, Radiation in a plasma, Physical Review CXII (1961) -711- V. L. GINzBUng, Flectomagnetic waves in a plasma, Pergamon Press Oxford (1964) \$12-13. D. Quexada, Ondes dans le plasma, Hermann Paris (1968) Cap. VI, 
$q$ la carica dell'elettrone, $\varepsilon$ e $\mu$ la costante dielettrica e la permeabilità del vuoto, $\boldsymbol{J}$ le correnti impresse che rappresentano le sorgenti del campo elettromagnetico.

Alle (1) e (2) si associano le equazioni:

$$
\frac{\partial N}{\partial t}+\operatorname{div}(N v)=0
$$

$$
N m \frac{d \boldsymbol{v}}{d t}=-\operatorname{grad} p+N q\left[\boldsymbol{E}+\mu_{\boldsymbol{v}} \times\left(\boldsymbol{H}_{0}+\boldsymbol{H}\right)\right]
$$

dove $m$ è la massa dell'elettrone, $H_{0}$ ¿̀ un campo magnetico esterno (cioè non dovuto alle correnti $\boldsymbol{J}+N q v$ della (1)) agente sul plasma, $p$ la pressione dovuta agli elettroni. Conforme agli antori sovra citati, e con ana riserva di cui diremo in seguito, ammetteremo $p$ scalare e solo funzione di $N$, cioè:

$$
p=f(N)
$$

Su $f(N)$ faremo, a seconda della questione in esame, una delle seguenti ipotesi che diremo, per brevita, a) o b):

a) Per ogni $N>0, f(N)$ è finita, continua, derivabile due volte con $f^{\prime}(N)$, $f^{\prime \prime}(N)$ finite e $f(N)>0, f^{\prime}(N)>0$.

b) Le proprietà della $f(N)$ sovra esposte valgono per $N \geq 0$ però, come ə̀ ovvio, $f(0)=0$.

È da notare che molti autori scrivono:

$$
f(N)=c N^{\gamma}
$$

con $c$ e $\gamma$ costanti positive. In questo caso l'ipotesi a) è verificata, ma per l'ipatesi b) occorre ammettere $\gamma=1$, ipotesi, questa, accolta in diverse trattazioni. L'ipotesi b) è perciò più restrittiva di $a$ ); per questa ragione abbiamo distinto il caso $N>0$ dal caso $N \geq 0$.

Ciò posto, passiamo ad enunciare un primo teorema di unicità.

Indicato con $\mathfrak{D}_{\infty}$ tutto lo spazio e con $(0, T)$ un intervallo finito di tempo ( $T$ finito e del resto arbitrario), una eventuale soluzione $\boldsymbol{E}, \boldsymbol{H}, \boldsymbol{N}, \boldsymbol{v}$ delle (1), $(2),(3),(4)$ e $(5)$ in $\mathfrak{D}_{0} \times(0, T)$ è nnioa purchè sia assegnato, in $\mathfrak{D}_{\infty} \times(0, T)$, $\boldsymbol{J}$ e $\boldsymbol{H}_{0}$ e, per $t=0, \boldsymbol{E}, \boldsymbol{H}, N, \boldsymbol{v}$ in tatto $\mathscr{D}_{\infty}$ (condizioni iniziali).

Il teorema verra provato nella classe delle funzioni $C^{(1)}$ (rispetto alle coordinate e al tempo), limitate in $\mathscr{D}_{\infty} \times(0, T)$, funzioni che, per brevità, diremo regolari, in base allipotesi $a)$ se $N>0$, in ogni dominio finito e per $t \in(0, T)$ e se $f^{\prime}(N)$ è limitata per ogni $N \geq 0$ limitato, oppure $N>0$ in $\left.\mathfrak{D}_{\infty} \times(0, T)\right)$; in base allipotesi b) se $N \geq 0$.

E bene notare, prima di proseguire, ehe la proprietà $N \geq 0$, per le solu- 
zioni delle equazioni ora considerate, è necessaria dal punto di vista fisico (supporre $N<0$ non ha senso). L'ipotesi $N>0$ si presenta, poi, abbastanza naturale in molti problemi fisici.

L'estensione del teorema di unicità al caso di un dominio finito $\mathfrak{D}$, anche ammesse le ipotesi a) e b) a seconda che $N>0, N \geq 0$ e le condizioni iniziali (in questo caso relative a $\mathfrak{D}$ ), presenta qualche difficoltà perchè, a quanto mi risulta, non vi è completo accordo, fra i cultori della teoria dei plasmi, sullo condizioni alla superficie che separa un plasma da un metallo o da un die. lettrico.

Noi faremo le seguenti tre ipotesi, sulla frontiera $\sigma$ di $\mathscr{D}$, indicando con $z$ un versore normale a $\sigma$ e orientato verso l'esterno di $\mathfrak{D}$.

I) - (ipotesi fluido-meccanica perchè analoga ad ipotesi che si possono fare nella teoria del moto dei flaidi). Per ogni $t \in(0, T)$, su $\sigma$ sono assegnati $\boldsymbol{v} \cdot \boldsymbol{z}$ e, dove $\boldsymbol{v} \cdot \boldsymbol{z}<0$, anche $\boldsymbol{v}$ e $N$. $t \in(0, T):$

II) - Su $\sigma$, che si suppone separi il plasma da un metallo, si ha, per

$$
v \cdot z=g(N)
$$

con $g(N)$ funzione positiva e crescente di $N$.

È da notare che la (6) comprende l'ipotesi (detta ipotesi di rigidità) $\boldsymbol{v} \cdot \boldsymbol{z}=0$, adottata da molti autori. $\left({ }^{2}\right)$

$$
\text { III) } \cdot \text { Su } \sigma \text { suppone } N=0 \cdot\left({ }^{3}\right)
$$

Naturalmente su $\sigma$ occorre assegnare condizioni per il campo elettromagnetico, ciod, al solito, la componente tangenziale di $\boldsymbol{E}$ o di $\boldsymbol{H}$.

Osserviamo che, in molti casi concreti, nella regione $\mathscr{R}$ occupata dalle correnti impresse (p. e. $\mathscr{R}$ è la regione occupata da un'antenna radio), $N=0$ o, meglio, il plasma occupa solo la regione esterna alla superficie $\sigma$ che limita

(2) Lijpotesi II si trova implicitamente nella memoria di R. H. WAIT, On the theory of wave propagation in a bounded plasma, Canadian Journal of Physics XLIV (1966) 293 Wait suppone v.z proporzionale, con coefficiente di proporzionalità positivo, a $p$ cioè a $f(N)$. Per le nostre ipotesi si ha subito (6).

(3) Questa ipotesi, nel caso di o metallica, ̀̀ ammessa da J. H. sics, John Wiley London (1968) Cap. V; $§ 3$.

Condizioni alla superficie che separa un metallo da un plasma sono state proposte anche da II H. СoнeN, Radiation in a plasma metal boundaries, Pysical Review CXXVI (1962) 369, condizioni però valide solo se il campo è sinoidale.

Per una discussione sulle propietà dei plagmi sulle pareli che lo limitano erf. M. P. BAchrnski, Sources in a mbounded plasma, Fifteenth General Assembly of U.R.S.I. Munich (1966) parte II, 1955. 
A. Allora, se su $\sigma$ valgono le ipotesi I, II, III, il teorema per $\mathscr{D}_{5} \times(0, T)$ rimane ancora valido senza ammettere assegnate su $\sigma$ le componenti di $E$ o di $\boldsymbol{H}$.

È bene infine osservare che, secondo alcuni autori, $\left(^{4}\right)$ il campo magnetico genera una anisotropia nella pressione degli elettroni, sicchè $p$ va sostituita da un tensore simmetrico $\pi$ (o un'omografia dilatazione) con la corrispondente $q u a d r i c a$ di rivoluzione rispetto a $\boldsymbol{H}_{0}$ e grad $p$ dalla divergenza di $\pi$. $\pi$ dipen. de da $\left(\boldsymbol{H}_{0}+\boldsymbol{H}\right) \Theta$ da $N$, però, poichè $\boldsymbol{H}$ è piccolo rispetto a $\boldsymbol{H}_{0}$, supporremo $\pi$ funzione solo di $N$ e $\boldsymbol{H}_{0}$ e, se $\boldsymbol{H}_{0}=0, \pi$ si riduca a $p$.

Però, in questo caso, sono riuseito a dimostrare il teorema di unicità solo per $N>0$ e sostituendo nella (4) alla derivata sostanziale la derivata lo. cale (il che è lecito solo se le velocità e le sue derivate rispetto alle coordi. nate sono molto piccole) e se le componenti di $\pi$, parallela e normale a $\boldsymbol{H}_{0}$, soddisfano all'ipotesi a).

Il procedimento per dimostrare i teoremi enunciati in questa introduzione è già stato usato da me $\mathrm{e}$ da altri autori in questioni di unicità per equazioni non lineari (5). Esso consiste nel provare, mediante il lemma di Gronwald, l'annullarsi dell'energia (o meglio un'espressione analoga) formata con la dif. ferenza di due eventuali soluzioni delle equazioni considerate. E bene notare che la principale difficoltà del procedimento consiste nel ridursi al lemma di GronwaLL.

2. - Riferendosi, per ora al caso, $p$ scalare, sostituendo (5) in (4) si ha:

$$
N m \frac{d v}{d t}=-f^{\prime}(N) \operatorname{grad} N+N q\left[\boldsymbol{E}+\mu v \times\left(\boldsymbol{H}_{0}+\boldsymbol{H}\right)\right]
$$

Ciò posto, per dimostrare il teorema di unicita, supporremo che (1), (2),

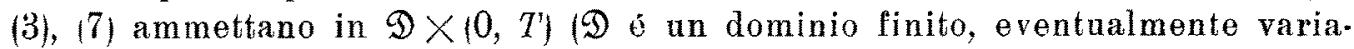
bile col tempol oltre che le soluzioni regolari $E, H, N, v$, le soluzioni, anch'esse regolari, $\boldsymbol{E}+\boldsymbol{e}, \boldsymbol{H}+\boldsymbol{h}, N+n, \boldsymbol{v}+\boldsymbol{w},(N+n) \geq 0$ in corrispondenza, si intende, delle stesse condizioni iniziali e con gli stessi valori di $\boldsymbol{J}$ e $\boldsymbol{H}_{0}$.

(4) Crf. Quemada, loco citato al n. 1.

(5) Crf. p.e. anche per la bibliografia, Sui teoremi di unicitò nella dinanica dei fluidi, Rendiconti del Seminario Mat. e Fis. di Milano XXXVI (1962). Per il lemma di Gronwall vedi G. SANsons, Equaziont differenziali nel campo reale, Zanichelli Bologna (196ỏ) p. 30. E bene notare ehe il procedimento accennato nel testo, per quanto mi risulta, fu applicato la prima volta da V. Yolterra, Sulle equazioni integro-differenziali, Rendiconti Lincei (5) XVIII (1909), 167, per equazioni integro-differenziali lineari Per equazioni differenziali non lineari (quelle dei fluidi incompressibili) ò stato applicato, in sostanza, la prima volta da E. EoA, Sullimpiego dell'analisi dimensionale nel moto turbolento, Lindustria XXXIII (1929). 
Sostituendo $\boldsymbol{E}+\boldsymbol{e}, \boldsymbol{H}+\boldsymbol{h}, \boldsymbol{N}+n, \boldsymbol{v}+\boldsymbol{w}$ nelle equazioni (1), (2), (3) e (7), tenendo conto che anche $\boldsymbol{E}, \boldsymbol{H}, N, \boldsymbol{v}$ soddisfano alle medesime equazioni, si ha, esplicitando la derivata sostanziale:

$$
\begin{gathered}
N m\left(\frac{\partial \boldsymbol{w}}{\partial t}+\frac{d(\boldsymbol{v}+\boldsymbol{w})}{d P} \boldsymbol{w}+\frac{d w}{d P} \boldsymbol{v}\right)+n m \frac{d(\boldsymbol{v}+\boldsymbol{w})}{d t}= \\
=-\left[f^{\prime}(N+n)-f^{\prime}(N)\right] \operatorname{grad}(N+n)-f^{\prime}(N) \operatorname{grad} n+q N[\boldsymbol{e}+ \\
\left.+\mu \boldsymbol{w} \times\left(H_{0}+\boldsymbol{H}\right)+\mu(\boldsymbol{v}+\boldsymbol{w}) \times \boldsymbol{h}\right]+q n\left[\boldsymbol{e}+\boldsymbol{E}+\mu(\boldsymbol{v}+\boldsymbol{w}) \times\left(\boldsymbol{H}_{0}+\boldsymbol{H}+\boldsymbol{h}\right)\right] \\
\frac{\partial \boldsymbol{n}}{\partial t}+\operatorname{div} N \boldsymbol{w}+\operatorname{div} n(\boldsymbol{v}+\boldsymbol{w})=0
\end{gathered}
$$

Converrà, ora, introdurre una ben conoseiuta e comoda notazione.

Siano $f(P, t)$ e $g(P, t)$ due funzioni (scalari o rettorali) di $P \in \mathscr{D}, t \in(0, T)$. Diremo che $g(P, t)=0(f(P, t))$ se si ha:

$$
|g(P, t)| \leq M \mid f(P, t): \quad \forall P \in \mathfrak{D}, t \in(0, T)
$$

dove $M$ indica un numero positivo.

Le proprietà del simbolo $O$ sono molto semplici e ben note; ricordiamo solo ehe, dalla nota disuguaglianza di $\mathrm{CAUCHY}_{\mathrm{U}}$, si deduce, se $f$ e $g$ sono scalari :

$$
f \cdot g=0\left(f^{2}\right)+O\left(g^{2}\right)
$$

e la stessa relazione vale se $f, g$ sono vettori intendendo per $f \cdot g$ il loro prodotto scalare in un punto generico $P \in \mathscr{D}$ e in un istante generico $t \in(0, T)$. Ciò premesso, si moltiplichi scalarmente la (8) per e e la (9) per $\boldsymbol{h}$ e poi si sottragga membro a membro, cioè si applichi a queste due equazioni il procedimento che conduce al teorema di Poynting.

Si ha allora, tenendo presente anche (12) e (13),

$$
\operatorname{div}(\boldsymbol{h} \times \boldsymbol{e})=\frac{1}{2} \frac{\partial}{\partial t}\left(\varepsilon e^{2}+\mu h^{2}\right)+O\left(e^{2}\right)+O\left(h^{2}\right)+O\left(N^{2} w^{2}\right)+O\left(n^{2}\right) .
$$

Passiamo, ora, alla (10) e osserviamo che, in $\mathfrak{D} \times(0, T)$, se $N>0$ vale l'ipotesi $a$ ) e $f^{\prime}(N)>0$, se $N \geq 0$ vale l'ipotesi $b$ ) ed $\delta$ ancora $f^{\prime}(N)>0$. Quindi 
è lecito dividere $(10)$ per $f^{\prime}(N)$ e $\frac{1}{f^{\prime}(N)}$ è limitata in $\mathfrak{D} \times(0, T)$.

Si ha poi, nello stesso dominio, per il teorema del valor medio, $0<\theta<1$

$$
f^{\prime}(N+n)-f^{\prime}(N)=f^{\prime \prime}(N+\theta n) n=O(n)
$$

Infatti, se $N>0,(N+n)>0,(N+\theta n) \dot{e}$, in $\mathscr{D} \times(0, T)$, limitata inferiormente da un numero positivo (il più piccolo fra i limiti inferiori di $N$ e $N+n$ ) percio, per l'ipotesi $a)$, anche $\left|f^{\prime \prime}(N+\theta n)\right| \ominus$ limitata; se $N \geq 0, N+n \geq 0$, vale l'ipotesi $b)$ e $\left|f^{\prime \prime}(N+\theta n)\right| \delta$ ancora limitata. Allora moltiplicando scalarmente la $(10)$, divisa per $f^{\prime}(N)$, per $N w$, ricordando in $\mathfrak{D} \times(0, T)$ limitate (perchè tali sono le derivate di $v) \frac{d(v+w)}{d P}, N, v, \frac{d(v+w)}{d t}$, ricordando la (13) e che $\frac{O(n)}{f^{\prime}(N)}$ ¿ ancora $O(n)$, si ha:

$$
\begin{gathered}
\frac{N^{2} m}{f^{\prime}(N)}\left(\frac{1}{2} \frac{\partial w^{2}}{\partial t}+\frac{d \boldsymbol{w}}{d P} v \cdot \approx v\right)=-N \operatorname{grad} n \cdot \boldsymbol{w}+\mathrm{O}\left(e^{2}\right)+\mathrm{O}\left(h^{2}\right)+ \\
+\frac{O\left(N^{2} \boldsymbol{w}^{2}\right)}{f^{\prime}(N)}+\mathrm{O}\left(n^{2}\right)
\end{gathered}
$$

Ora si osservi che:

$$
N \operatorname{grad} n \cdot w=\operatorname{div}(N n w)-n \operatorname{div}(N w)
$$

Si ha poi, indicando con $K \frac{d \boldsymbol{w}}{d P}$ l'omografia coniugata (o trasposta) di $\frac{d w}{d P}$ :

$$
\begin{aligned}
& \frac{N^{2}}{f^{\prime}(N)} \frac{d \boldsymbol{w}}{d P} \boldsymbol{v} \cdot \boldsymbol{w}=\frac{N^{2}}{f^{\prime}(N)} K \frac{d \boldsymbol{w}}{d P} \boldsymbol{w} \cdot v=\frac{1}{2} \operatorname{grad} \boldsymbol{w}^{2} \cdot \frac{N^{2}}{f^{\prime}(N)} v= \\
& =\frac{1}{2} \operatorname{div}\left(\frac{N^{2} w^{2}}{f^{\prime}(N)} v\right)-\frac{1}{2} \operatorname{grad} \frac{N^{2}}{f^{\prime}(N)} \cdot v w^{2}-\frac{1}{2} \frac{N^{2} w^{2}}{f^{\prime}(N)} \operatorname{div} v
\end{aligned}
$$

Si ha poi:

$$
\frac{1}{2} \frac{N^{2}}{f^{\prime}(N)} \frac{\partial w^{2}}{\partial t}=\frac{1}{2} \frac{\partial}{\partial t}\left(\frac{N^{2}}{f^{\prime}(N)} w w^{2}\right)-\frac{1}{2} \frac{\partial}{\partial t}\left(\frac{N^{2}}{f^{\prime}(N)}\right) w^{2}
$$

quindi :

$$
\begin{gathered}
\frac{N^{2}}{f^{\prime}(N)}\left(\frac{1}{2} \frac{\partial w^{2}}{\partial t}+\frac{d w}{d P} v \cdot w\right)=\frac{1}{2} \frac{\partial}{\partial t}\left(\frac{N^{2}}{f^{\prime}(N)} w^{2}\right)+ \\
+\frac{1}{2} \operatorname{div}\left(\frac{N^{2} w^{2}}{f^{\prime}(N)} v\right)-\frac{1}{2}\left[\frac{\partial}{\partial t} \frac{N^{2}}{f^{\prime}(N)}+\operatorname{grad} \frac{N^{2}}{f^{\prime}(N)} \cdot v\right] w^{2}-\frac{1}{2} \frac{N^{2} w^{2}}{f^{\prime}(N)} \operatorname{div} v
\end{gathered}
$$


Ora, ricordando (3) in eni conviene sviluppare div $(N v)$; si ha

$$
\begin{gathered}
\frac{\partial}{\partial t} \frac{N^{2}}{f^{\prime}(N)}+\operatorname{grad} \frac{N^{2}}{f^{\prime}(N)} \cdot v=\frac{d}{d N}\left(\frac{N^{2}}{f^{\prime}(N)}\right)\left(\frac{\partial N}{\partial t}+\operatorname{grad} N \cdot v\right)= \\
=-\frac{2 f^{\prime}(N) N-f^{\prime \prime}(N) N^{2}}{\left(f^{\prime}(N)\right)^{2}} N \operatorname{div} v
\end{gathered}
$$

Tenendo presente che $N$ e $\frac{1}{f^{\prime}(N)}$ sono limitati in $\mathfrak{D} \times(0, T)$, si conclude che la somma degli ultimi tre termini di $(17)$ è $O\left(\frac{N^{2} w^{2}}{f^{f}(N)}\right)$.

Allora, tenendo conto anche di (16), la (15) diventa:

$$
\begin{aligned}
\frac{m}{2} \frac{\partial}{\partial t}\left(\frac{N^{2}}{f^{\prime}(N)} w^{2}\right)+\frac{m}{2} & \operatorname{div}\left(\frac{N^{2}}{f^{\prime}(N)} w^{2} v\right)+\operatorname{div}(N n w)-n \operatorname{div}(N w)= \\
= & 0\left(e^{2}+h^{2}+\frac{N^{2}}{f^{\prime}(N)} w^{2}+n^{2}\right) .
\end{aligned}
$$

Moltiplichiamo infine (11) per $n$. Tenendo presente che:

$n \operatorname{div}[n(v+w)]=n \operatorname{grad} n \cdot(v+w)+n^{2} \operatorname{dịv}(v+w)=$

$=\frac{1}{2} \operatorname{grad} n^{2} \cdot(v+w)+n^{2} \operatorname{div}(v+w)=\frac{1}{2} \operatorname{div}\left[n^{2}(v+w)\right]+\frac{1}{2} n^{2} \operatorname{div}(v+w)$

si ha, sempre in $\mathscr{D} \times(0, T)$ :

$$
\frac{1}{2}\left[\frac{\partial}{\partial t} n^{2}+\operatorname{div}\left(n^{2}(v+w)\right)\right]+n \operatorname{div}(N w)=O\left(n^{2}\right)
$$

Allora, sommando (14), (18), (19), tenendo sempre presente che $\frac{1}{f^{\prime}(N)}$ è limitata sicchè $N^{2} w^{2}=O\left(\frac{N^{2} w^{2}}{f^{\prime}(N)}\right)$ e inoltre che $O\left(e^{2}\right)$ e $O\left(h^{2}\right)$ equivalgono a $O\left(\varepsilon e^{2}\right)$ e $\mathrm{O}\left(\mu h^{2}\right)$, si ha:

$$
\begin{gathered}
\frac{\partial}{\partial t}\left(\varepsilon e^{2}+\mu h^{2}+\frac{N^{2}}{f^{\prime}(N)} m w^{2}+n^{2}\right)+\operatorname{div}\left[2 \boldsymbol{e} \times \boldsymbol{h}+\frac{m N^{2}}{f^{\prime}(N)} w^{2} v+2 N n \boldsymbol{w}+\right. \\
\left.+n^{2}(\boldsymbol{v}+\boldsymbol{w})\right]=\mathrm{O}\left(\varepsilon e^{2}+\mu h^{2}+\frac{m N^{2}}{f^{\prime}(N)} w^{2}+n^{2}\right) .
\end{gathered}
$$


Converrà, per il seguito, porre:

$$
a=\varepsilon e^{2}+\mu h^{2}+\frac{m N^{2}}{f^{\prime}(N)} w^{2}+n^{2}
$$

sicchè, ricordando la definizione del simbolo $O$, si potrà anche scrivere:

$$
\frac{\partial a}{\partial t}+\operatorname{div}\left[2 \boldsymbol{e} \times \boldsymbol{h}+\frac{m N^{2}}{f^{\prime}(N)} w^{2} v+n^{2}(\boldsymbol{v}+\boldsymbol{w})+2 N n \boldsymbol{w}\right] \leq M a
$$

3 - Passiamo, ora, a dimostrare il teorema di unicità, nel caso del dominio illimitato.

Sia $c$ un numero tale da soddisfare, in tutto $\mathfrak{D}_{\infty} \times(0, T)$, alle relazioni:

$$
\begin{gathered}
c>\frac{1}{\sqrt{\varepsilon \mu}}, \\
c>|v|+|w|+\sqrt{\frac{f^{\prime}(N)}{m}}
\end{gathered}
$$

Sia ora $P_{0}$ un punto generico dello spazio, $\Sigma(t)$ una superficie sferica di centro $P_{0} \otimes$ che va contraendosi al trascorrere del tempo, più precisamente con raggio $R$ tale ohe $R=R_{0}-c t, R_{0}>c T$, sicchè la sfera, nell'intervallo $(0, T)$, non si annulla mai; assumeremo questa sfera come il dominio $\mathscr{D}$ considerato nel numero precedente.

Perciò, in ogni punto del volume $S(t)$ interno a $\Sigma(t)$, vale la (22) e, integrando questa equazione su $S(t)$, ricordando la formula (per semplicità tipografica scriveremo $\Sigma$ e $S$ invece di $\Sigma(t)$ e $S(t))$ :

$$
\frac{d}{d t} \int_{S} a d S=\int_{S} \frac{\partial a}{\partial t} d S-c \int_{\Sigma} a d \Sigma
$$

si ha, se $r$ è il versore unitario normale a $\Sigma$ e diretto verso l'esterno di $S$ :

$$
\begin{gathered}
\frac{d}{d t} \int_{S} a d S+\int_{\Sigma}\left[c a+\left(2 \boldsymbol{e} \times \boldsymbol{h}+\frac{m N^{2}}{f^{\prime}(N)} w^{2} \boldsymbol{v}+n^{2}(\boldsymbol{v}+\boldsymbol{w})+2 N n \boldsymbol{w}\right) \cdot \boldsymbol{r}\right] d \Sigma \leq \\
\leq M \int_{S} a d S
\end{gathered}
$$


Ora, ricordando (23), $\left(23^{\prime}\right)$ e (21):

$$
\begin{gathered}
c a+\left(2 \boldsymbol{e} \times \boldsymbol{h}+\frac{m N^{2}}{f^{\prime}(N)} w^{2} \boldsymbol{v}+2 N n \boldsymbol{w}+n^{2}(\boldsymbol{v}+w)\right) \cdot \boldsymbol{r} \geq \\
\geq\left(\sqrt{\frac{\varepsilon}{\mu}} e^{2}+\sqrt{\frac{\mu}{\varepsilon}} h^{2}-2|\boldsymbol{e} \times \boldsymbol{h}|\right)+\left(c-|\boldsymbol{v}|-\sqrt{\frac{f^{\prime}(N)}{m}}\right) \frac{m N^{2}}{f^{\prime}(N)} w^{2}+\sqrt{\frac{m}{f^{\prime}(N)}} N^{2} w^{2}+ \\
+\left(c-|\boldsymbol{v}|-|\boldsymbol{w}|\left|n^{2}-2 N n\right| \boldsymbol{w} \mid \geq\right. \\
\geq\left(\sqrt{\frac{\varepsilon}{\mu}} \boldsymbol{e}-\sqrt{\frac{\mu}{\varepsilon}}\right)^{2}+\left(c-|\boldsymbol{v}|-\sqrt{\frac{f^{\prime}(N)}{m}}\right) \frac{m N^{2}}{f^{\prime}(N)} w^{2}+ \\
+\left(\sqrt{\frac{m}{f^{\prime}(N)}} N|\boldsymbol{w}|-\sqrt{\frac{f^{\prime}(N)}{m}} n\right)^{2}>0
\end{gathered}
$$

Quindi :

$$
\frac{d}{d t} \int_{S} a d S \leq M \int_{S} a d S
$$

Ora, poichè per le condizioni iniziali $a \equiv 0$ per $t=0$, per il lemma di Grontalu si ha, in ogni istante, $\int_{S} a d S=0$ e, poichè $a \geq 0$, si ha $a(t) \equiv 0$ in tatti $i$ punti interni alla superficie sferica $\Sigma(t)$.

Poiché $P_{0}$ è interno a $\Sigma(t)$ per $t \in(0, T)$ si ha, in questo intervallo, $a \equiv 0$ in $P_{0}$. Quindi, poiché per (21) a é una somma di termini positiva o nulli si ha, in $P_{0}$ e per $t \in(0, T), \boldsymbol{e}=\boldsymbol{h}=\boldsymbol{w}=0, n=0$.

Ora, $P_{0}$ è un punto qualunque dello spazio, dunque il teorema di unicità è provato. $\left({ }^{6}\right)$

4. - Passiamo, ora, al caso in cui il plasma occupa un dominio finito invariabile $\mathscr{D}$, limitato dalla superficie $\sigma$.

Se $S$ è il volume del dominio e se $z$ è il versore normale a $\sigma$, diretto verso l'esterno di $S$, dalla (20), tenendo presente (22), si ha:

$$
\begin{aligned}
\frac{d}{d t} \int_{S} a d S+\int_{\sigma}[2(\boldsymbol{e} \times \boldsymbol{h}) \cdot \boldsymbol{z} & \left.+\frac{m N^{2}}{f^{\prime}(N)} \boldsymbol{w}^{2} \boldsymbol{v} \cdot \boldsymbol{z}+n^{2}(\boldsymbol{v}+\boldsymbol{w}) \cdot \boldsymbol{z}+2 N n_{\boldsymbol{w}} \cdot \boldsymbol{z}\right] d \sigma \leq \\
& \leq M \int_{S} a d S
\end{aligned}
$$

(6) $\mathrm{E}$ bene notare che la condizione $a \equiv 0$ impliea $N w=0$ quindi, a rigore, $w=0$ solo nell'insieme contenuto in $\mathfrak{D}_{\infty} \times(0, T)$ in cui $N \neq 0$. Se questo insieme è di misura nulla, $n=0$ ovunque per continuită, comunque dove $N=0$ la velocita dei corpuscoli non ha significato fisico, 
Ora, nelle ipotesi del n. 1, l'integrale esteso a $\sigma$ è positivo a nullo.

Per provare eiò, si osservi anzitutto che su $\sigma$ sono assegnate le componen. ti tangenziali di $\boldsymbol{E}$ o di $\boldsymbol{H}$ quindi sono nulle le componenti tangenziali di $\boldsymbol{e}$ o di $\boldsymbol{h}$; il primo termine dell'integrale esteso a $\sigma$ è perciò nullo.

Ora, nell'ipotesi I (caso fluido-dinamicol é assegnata la componente normale a $\sigma$ di $v, v \cdot z$, quindi $\boldsymbol{w} \cdot \boldsymbol{z}=0$; l'ultimo termine dell'integrale in discorso è dunque nullo. Inoltre, in questo caso, nei punti di $\sigma$ dove $v \cdot z<0, v$ e $N$ sono assegnati sicchè, in questi punti, $w=0$ e $n=0$; restano perciò, nel l'integrale esteso a $\sigma$, solo termini non negativi, esso è danque positivo o nullo.

Nel caso (ipotesi II) $\boldsymbol{v} \cdot \boldsymbol{z}=g(N) \geq 0$ e quindi $(\boldsymbol{v}+0) \cdot z=g(N+n) \geq 0$, si ha che il secondo e terzo termine, nell'integrale esteso a $\sigma$, non sono negativi l'ultimo termine vale, poi, $2 N(g(N+n)-g(N)) n$ che non può essere negativo perchè $g(N)$ è crescente. Quindi, anche in questo caso, l'integrale esteso a $\sigma$ è positivo o nullo.

Se, infine, il numero degli elettroni su $\sigma$ è sempre nullo (ipotesi III), si ha $N=0, n=0$ e l'integrale esteso a $\sigma$ è nullo.

In ogni caso, dunque, (27) si riduce a (26), percio si ha (essendo $a=0$ per $t=0$, in conseguenza delle condizioni iniziali) per ogni $P \in S$ e ogni $t \in(0, T), a \equiv 0$, cioé (con la riserva di nota (6)) $\boldsymbol{e}=\boldsymbol{h}=\boldsymbol{w}=0, n=0$ e il teorema di unicità risulta anche in questo caso provato.

5. - Sia ora $\sigma$ la superficie (finita) che limita le sorgenti; il plasma occupi tutta e solo la regione dello spazio esterna a $\sigma . \theta$ in questa regione e su $\sigma$, soddisfi alle ipotesi del n. 1.

Sia ancora, come al n. $3, P_{0}$ un punto dello spazio esterno a $\sigma,{ }^{5} R$ il raggio della sfera $\Sigma(t)$ e $R_{0}$ sia scelto in modo che $\Sigma(t)$ contenga nel proprio interno $\sigma$, in tutto l'intervallo di tempo $(0, T)$.

Ora, nei punti del volume $S_{1}(t)$, compreso fra $\Sigma(t)$ e $\sigma$, vale la $(20)$; integrando questa equazione su $S_{1}(t)$ e ragionando come al $n .3$, si ottiene la (27) parchè, in luogo di $S$, si sostituisca $S_{1}$. E, ragionando come al $n .4$, si ha che gli altimi tre termini dell'integrale esteso a $\sigma$ sono positivi o nulli, quindi:

$$
\frac{d}{d t} \int_{S_{1}} \alpha d S_{1} \leq 2 \int_{\sigma}(\boldsymbol{e} \times \boldsymbol{h}) \cdot \boldsymbol{z} d \sigma+M \int_{S_{1}} a d S_{1}
$$

Il volume $S_{2}$, interno a $\sigma$, non è occupato da plasma, perciò si può porre per (21)

$$
a=\varepsilon e^{2}+\mu h^{2}
$$

e da (7) e (8) considerate in $S_{2}$, sicchè si deve porre $N=0, n=0$, con il ben 
noto procedimento con cui si ginnge al teorema di PoynTING, si ottiene:

$$
\frac{d}{d t} \int_{S_{2}} a d S_{2}=-2 \int_{\sigma}(\boldsymbol{e} \times \boldsymbol{h}) \cdot \boldsymbol{z} d \sigma
$$

Sommando (29) e (30) si ricava subito (26) e perciò, ragionando nel solito modo, il teorema di unicita.

6. - Passiamo ora ad accennare al caso in cui la pressione sia rappresentata da un tensore $\pi\left(N, \boldsymbol{H}_{0}\right)$. Poichè, come si è detto nell'introduzione, la quadrica relativa a $\pi$ è di rivoluzione rispetto a $\boldsymbol{H}_{0}$, esso sarà determinato, assegnate le sue componenti $f_{i}\left(\boldsymbol{H}_{0}, N\right), f_{1}\left(\boldsymbol{H}_{0}, N\right)$ parallela e perpendicolare a $\boldsymbol{H}_{0}$. Ammetteremo $f_{i}\left(\boldsymbol{H}_{0}, N\right), f_{1}\left(\boldsymbol{H}_{0}, N\right)$ soddisfacenti alle medesime proprietà della $f(N)$ considerata nel primo paragrafo; in particolare le componenti di $\frac{\partial \pi}{\partial N}$ saranno $\frac{\partial f_{\|}}{\partial N}\left(N, \boldsymbol{H}_{0}\right), \frac{\partial f_{\perp}}{\partial N}\left(N, H_{0}\right)$ (o, più brevemente, $\left.f_{\|}(N), f_{\perp}^{\prime}(N)\right)$ diverse da zero, e perciò, esiste $\left(\frac{\partial \pi}{\partial N}\right)^{-1}$ con componenti parallela e rispettivamente a $\frac{1}{f_{\mathrm{l}}^{\prime}(N)}, \frac{1}{f_{1}^{\prime}(N)}$, quindi in particolare:

$$
\left(\frac{\partial \pi}{\partial N}\right)^{-1} w \cdot w=\frac{w_{1}^{2}}{f^{\prime}(N)}+\frac{w_{\perp}^{2}}{f_{\perp}^{\prime}(N)}
$$

se $w_{\|}$e $w_{1}$ sono le componenti di $\boldsymbol{w}$ rispettivamente parallela e normale a $\boldsymbol{H}_{0}$.

Ammetteremo, inoltre, limitate e lipschitziane rispetto a $N$ le derivate rispetto a $\boldsymbol{H}_{0}$ delle componenti di $\pi$.

Come si 6 accennato, ci limiteremo a dimostrare il teorema nel caso in cui la derivata sostanziale $\frac{d v}{d t}$ venga sostituita dalla derivata locale $\frac{\partial v}{d t} \mathrm{e}$ $\operatorname{sia} N>0$.

Allora restano ancora valide (8), (9), (11) e le loro conseguenze (14), (19); la (10) viene però modificata nel seguente modo (indicheremo, conforme a simboli introdotti da Bunali Forti e Marcolongo, con grad $\pi$ la divergenza di $\pi)$ :

$$
n m \frac{\partial(\boldsymbol{v}+\boldsymbol{w})}{\partial t}+N m \frac{\partial \boldsymbol{w}}{\partial t}=-\operatorname{grad}\left[\pi\left(N+n, \boldsymbol{H}_{0}\right)-\pi\left(N, \boldsymbol{H}_{0}\right)\right]+
$$

$+N q\left[\boldsymbol{e}+\mu z \times\left(\boldsymbol{H}_{0}+\boldsymbol{H}\right)+\mu(\boldsymbol{v}+\boldsymbol{w}) \times \boldsymbol{h}\right]+n q\left[\boldsymbol{E}+\boldsymbol{e}+\mu(\boldsymbol{v}+\boldsymbol{w}) \times\left(\boldsymbol{H}_{0}+\boldsymbol{H}+\boldsymbol{h}\right)\right]$. 
Ora, è noto (7) (il significato dei simboli é ovvio) che:

$$
\begin{gathered}
\operatorname{grad} \pi\left(N, \boldsymbol{H}_{0}\right)=\frac{\partial(\pi \boldsymbol{i})}{\partial x}+\frac{\partial(\pi \boldsymbol{j})}{\partial y}+\frac{\partial(\pi \boldsymbol{k})}{\partial z}= \\
=\frac{\partial \pi}{\partial N} \operatorname{grad} N+\frac{\partial(\pi \boldsymbol{i})}{\partial \boldsymbol{H}_{0}} \cdot \frac{\partial \boldsymbol{H}_{0}}{\partial x}+\frac{\partial(\pi \boldsymbol{j})}{\partial \boldsymbol{H}_{0}} \cdot \frac{\partial \boldsymbol{H}_{0}}{\partial y}+\frac{\partial(\pi \boldsymbol{k})}{\partial \boldsymbol{H}_{0}} \cdot \frac{\partial \boldsymbol{H}_{0}}{\partial z}
\end{gathered}
$$

Quindi, per le nostre ipotesi abbiamo, come al n. 2 , in $\mathfrak{D} \times(0, T)$ :

$$
\operatorname{grad} \pi\left(N+n, \boldsymbol{H}_{0}\right)-\operatorname{grad} \pi\left(N, \boldsymbol{H}_{0}\right)=\frac{\partial \pi}{\partial N} \operatorname{grad} n+O(n) .
$$

Ora, moltiplicando (31) per $N\left(\frac{\partial \pi}{\partial N}\right)^{-1} w$ otteniamo, ricordando poi che, essendo $N>0,\left(\frac{\partial \pi}{\partial N}\right)^{-1}$ è limitata in $\mathscr{D} \times\left(0, T^{\prime}\right)$ :

$$
N^{2} m\left(\frac{\partial \pi}{\partial N}\right)^{-1} \boldsymbol{w} \cdot \frac{\partial \boldsymbol{w}}{\partial t}=-N \operatorname{grad} n \cdot \boldsymbol{w}+O\left(N^{2} w^{2}\right)+O\left(n^{2}\right)+O\left(e^{2}\right)+O\left(h^{2}\right)
$$

che si prò anche scrivere, poichè $\left(\frac{\partial \pi}{\partial N}\right)^{-1}$ è simmetrica,

$$
\begin{gathered}
\frac{m}{2} \frac{\partial}{\partial t}\left[N^{2}\left(\frac{\partial \pi}{\partial N}\right)^{-1} \boldsymbol{w} \cdot \boldsymbol{w}\right]-\frac{m}{2} \frac{\partial}{\partial t}\left(N^{2} \frac{\partial \pi}{\partial N}\right)^{-1} \boldsymbol{w} \cdot \boldsymbol{w}= \\
=-\operatorname{div}(N n \boldsymbol{w})+n \operatorname{div}(N \boldsymbol{w})+O\left(N^{2} w^{2}\right)+O\left(n^{2}\right)+O\left(e^{2}\right)^{2}+O\left(h^{2}\right)
\end{gathered}
$$

Ora, poiche in $\mathfrak{D} \times(0, T)$ é $N>0$, l'ultimo termine a primo membro si puo moltiplicare e dividere per $N$ e risulta del tipo $O\left(N^{2} w^{2}\right)$ di modo che, trasportato al secondo membro, si può conglobare nell'altro termine dello stesso ordine.

Allora, sommando (33) con (18) e (19), posto

$$
a=\varepsilon e^{2}+\mu h^{2}+m N^{2}\left(\frac{\partial \pi}{\partial N}\right)^{-1} \boldsymbol{w} \cdot \boldsymbol{w}+n^{2}
$$

(7) La (32) si può giustificare nel seguente modo. Posto $i_{1}=i, i_{2}=j, i_{3}=k$ si ha (si ricordi che il tensore è simmetrico e, al solito, si sottointende la sommatoria rispetto agli indici $r \in j$ )

$$
\operatorname{grad} \pi=\frac{\partial p_{r j}}{\partial x_{j}}{ }^{r}=\frac{\partial\left(p_{r j} i_{i}\right)}{\partial x_{j}}=\frac{\partial\left(\pi i_{j}\right)}{\partial x_{j}}
$$

conforme al testo, 
considerando, come al n. 3 , la sfera $\Sigma(t)$ di centro $P_{0}$ e raggio $R=R_{0}-c t$, $\left(R_{0}-c t\right)>0$, e ragionando come al numero citato $\theta$ con gli stessi simboli, si ha:

$$
\frac{d}{d t} \int_{S} a d S+\int_{\Sigma}\left[c a+\left(2 \boldsymbol{e} \times \boldsymbol{h}+n^{2}(\boldsymbol{v}+\boldsymbol{w})+2 N n \boldsymbol{w}\right) \cdot \boldsymbol{r}\right] d \Sigma \leq M \int_{S} a d S
$$

Ora $c$ verrà supposta soddisfacente, per $|\boldsymbol{v}|,|\boldsymbol{w}|, N$ in $\mathscr{D}_{\infty} \times(0, T)$, alla (23) e alle relazioni:

$$
\begin{aligned}
& c>|v|+|\boldsymbol{w}|+\sqrt{\frac{\overline{f_{\|}^{\prime}(N)}}{m}}, \\
& c>|v|+|\boldsymbol{w}|+\sqrt{\frac{f_{1}^{\prime}(N)}{m}}
\end{aligned}
$$

Supposto, per fissare le idee, che nel punto generico $P$ di $\Sigma$ e all'istante $t$, sia $f_{\mid}^{\prime}>f_{\perp}^{\prime}$, si ha, ricordando anche qualche risultato del n. 3 :

$$
\begin{gathered}
c a+\left[2 \mathbf{e} \times \boldsymbol{h}+n^{2}(\boldsymbol{v}+\boldsymbol{w})+2 n N \boldsymbol{w}\right] \cdot \boldsymbol{r}>\left(\sqrt[4]{\frac{\varepsilon}{\mu}}-\sqrt[4]{\frac{\mu}{\varepsilon}} \boldsymbol{h}\right)^{2}+ \\
+N^{2} m \sqrt{\frac{f_{\|}^{\prime}}{m}}\left(\frac{w_{\|}^{2}}{f_{\|}^{\prime}}+\frac{w_{1}^{2}}{f_{1}^{\prime}}\right)+\left(c-|\boldsymbol{v}|-|\boldsymbol{w}|-\sqrt{\frac{f_{\|}^{\prime}}{m}}\right) n^{2}+\sqrt{\frac{f_{\|}^{\prime}}{m}} n^{2}-2 n N|\boldsymbol{w}|> \\
>N^{2} w^{2} \sqrt{\frac{m}{f_{\|}}}+\sqrt{\frac{f_{\|}^{\prime}}{m}} n^{2}-2 n N|\boldsymbol{w}|= \\
=\left(\sqrt{\frac{m}{f_{\|}^{\prime}}} N|\boldsymbol{w}|-\sqrt{\frac{f_{\|}^{\prime}}{m}} n\right)^{2} \geq 0
\end{gathered}
$$

e allo stesso risultato si giunge se $f_{\perp}^{\prime}>f_{\|}^{\prime}$.

Allora la (35) si riduce a (27) $\theta$, poichè $\alpha \geq 0$, ragionando come al n. 3, si deduce subito il teorema di unicità nel caso dello spazio illimitato.

Considerazioni analoghe al n. 4 potrebbero svilupparsi nel caso del dominio limitato, ma su ciò non insisteremo.

7. - Osserveremo, da ultimo, tornando al caso in cui la pressione si riduce a uno scalare, che il teorema di unicità si poteva anche ottenere senza dividere la $(10)$ per $f^{\prime}(N)$, moltiplicando però per $f^{\prime}(N)$ la (11); comunque senza alcuna modifica delle ipotesi del n. 1 .

Questo procedimento, però, non mi sembra facilmente applicabile al caso in cui la pressione è un tensore. 